\title{
On the effects of rotation in primordial star-forming clouds
}

\author{
Jayanta Dutta ${ }^{1,2}$ \\ ${ }^{1}$ Instituto de Astrofisica e Ciencias do Espaco, Universidade de Lisboa, OAL, Tapada da Ajuda, PT1349-018 Lisboa, Portugal
e-mail: jd. astrop@gmail.com
${ }^{2}$ Inter-University Centre for Astronomy and Astrophysics, Post Bag 4, Ganeshkhind, Pune University Campus, 411007 Pune, India \\ Received 15 June 2015 / Accepted 13 October 2015
}

\begin{abstract}
Context. The thermodynamical evolution of gas during the collapse of the primordial star-forming cloud depends significantly on the initial degree of rotation.

Aims. However, there is no clear understanding of how the initial rotation can affect the heating and cooling process and hence the temperature that leads to the fragmentation of the gas during Population III star formation.

Methods. We report the results from three-dimensional, smoothed-particle hydrodynamics (SPH) simulations of a rotating selfgravitating primordial gas cloud with a modified version of the Gadget-2 code, in which the initial ratio of the rotational to the gravitational energy $\left(\beta_{0}\right)$ is varied over two orders of magnitude.

Results. We find that despite the lack of any initial turbulence and magnetic fields in the clouds, the angular momentum distribution leads to the formation and build-up of a disk that fragments into several clumps. We further examine the behavior of the protostars that form in both idealized as well as more realistic minihalos from the cosmological simulations. The thermodynamical evolution and the fragmentation behavior of the cosmological minihalos are similar to that of the artificial cases, especially in those with a similar $\beta_{0}$-parameter. Protostars with a higher rotation support exhibit spiral-arm-like structures on several scales, and have lower accretion rates. These type of clouds tend to fragment more, while some of the protostars escape from the cluster with the possibility of surviving until the present day. They also take much longer to form compared to their slowly rotating counterparts.

Conclusions. We conclude that the use of appropriate initial conditions of the gas in minihalos is a pivotal and decisive quantity to study the evolution and final fate of the primordial stars.
\end{abstract}

Key words. hydrodynamics - instabilities - early Universe - stars: Population III - stars: formation

\section{Introduction}

Since the late 1960s, the theoretical study of the Universe at high redshift has persuaded a number of groups to work persistently on the dynamics of collapsing gas clouds (Saslaw \& Zipoy 1967; Peebles \& Dicke 1968; Silk 1977; Couchman \& Rees 1986; Haiman et al. 1996; Tegmark et al. 1997), leading to the formation of the very first stars in the Universe, the so-called Population III (or Pop III) stars. Based on these leading-edge studies, the first sources of light are believed to have formed only a few hundred million years after the Big Bang (Barkana \& Loeb 2001; Bromm \& Larson 2004; Ciardi $\&$ Ferrara 2005). This era marks a crucial transition from the simple to the complex Universe (Loeb 2010; Bromm \& Yoshida 2011; Glover 2013). Subsequent pioneering three-dimensional numerical simulations using adaptive mesh refinement (Abel et al. 2000; O'Shea \& Norman 2006), smoothed-particle hydrodynamics (Yoshida et al. 2008) or more recently using the hydrodynamic moving mesh code Arepo (Greif et al. 2011, 2012) have led to the development of a widely accepted standard model of primordial star formation. In this star formation model, the first protostars form within dark matter (DM) halos with a virial temperature of $\sim 1000 \mathrm{~K}$ and masses of $\sim 10^{5}-10^{6} M_{\odot}$, which had collapsed at redshift $z \geq 20$. However, other simulations show that Pop III stars may still form well beyond this, in waves that delay their formation (e.g., Scannapieco et al. 2003; Tornatore et al. 2007; Ritter et al. 2012). These studies suggest that Pop III star can continue to form down to the redshift $z=2.5$ with a low peak rate of $10^{-5} M_{\odot} \mathrm{yr}^{-1} \mathrm{Mpc}^{-3}$, which occurred at $z=6$. Observational evidence of Pop III stars and the possibility of Pop III waves reaching lower redshifts has received a significant boost with the discovery of a luminous Lyman- $\alpha$ (Ly $\alpha)$ emitter with high EW He II and Ly $\alpha$ emission and no metal lines (Sobral et al. 2015).

The hydrogen atoms combine with the free electron (present from the epoch of recombination at $z \sim 1100$ ) to produce small fractional abundances of $\mathrm{H}_{2}, x_{\mathrm{H}_{2}} \sim 10^{-3}$ (Susa et al. 1998; Yoshida et al. 2003). As the collapse proceeds, the gas is cooled via $\mathrm{H}_{2}$ rotational and vibrational line emission (also termed as gas-phase reaction), with $\mathrm{H}^{-}$ion as an intermediate state (first discussed in the context of the local ISM by McDowell 1961)

$\mathrm{H}+\mathrm{e}^{-} \rightarrow \mathrm{H}^{-}+\gamma$,

$\mathrm{H}^{-}+\mathrm{H} \rightarrow \mathrm{H}_{2}+\mathrm{e}^{-}$,

where the free electrons act as catalysts (Omukai \& Nishi 1998). At this point, gas attains a temperature $\sim 200 \mathrm{~K}$, and is in local thermodynamical equilibrium (LTE) with the kinetic temperature of the gas. However, the limited $\mathrm{H}_{2}$ abundance is not sufficient to cool the gas further, and the gas begins to heat up with increasing density. This results in the free-fall time being shorter than the cooling time. The transition from the cooling (low density) to heating (high density) with increasing density occurs near a critical density $n_{\mathrm{cr}} \approx 10^{4} \mathrm{~cm}^{-3}$, and sets a characteristic Jeans length, allowing the gas to fragment with the Jeans mass of $M_{\mathrm{J}}\left(200 \mathrm{~K}, 10^{4} \mathrm{~cm}^{-3}\right) \sim 1000 M_{\odot}$ (Abel et al. 2002). At 
higher density $\left(\sim 10^{8} \mathrm{~cm}^{-3}\right)$ hydrogen molecules are formed by the three-body reactions (Palla et al. 1983)

$\mathrm{H}+\mathrm{H}+\mathrm{H} \rightarrow \mathrm{H}_{2}+\mathrm{H}$

$\mathrm{H}+\mathrm{H}+\mathrm{H}_{2} \rightarrow \mathrm{H}_{2}+\mathrm{H}_{2}$.

There is however a significant uncertainty in the rate coefficients (Glover \& Savin 2009) for the above set of reactions that can cool the gas rapidly by converting almost all the atomic hydrogen into molecules, making the gas chemothermally unstable (Turk et al. 2009; Dutta 2015a).

However, the amount of hydrogen molecules produced is a strong function of temperature. At high temperature, $\mathrm{H}_{2}$ molecules are destroyed by collisions with atomic $\mathrm{H}$ and $\mathrm{H}_{2}$ molecules (Yoshida et al. 2006),

$\mathrm{H}_{2}+\mathrm{H} \rightarrow \mathrm{H}+\mathrm{H}+\mathrm{H}$,

$\mathrm{H}_{2}+\mathrm{H}_{2} \rightarrow \mathrm{H}+\mathrm{H}+\mathrm{H}_{2}$.

The collision dissociation of $\mathrm{H}_{2}$ prevents the fractional abundance $\left(x_{\mathrm{H}_{2}}\right)$ from becoming large. The study by Turk et al. (2011) has discussed the equilibrium condition between the abundances of atomic and molecular hydrogen, known as the principle of microscopic reversibility. Therefore the rate at which $\mathrm{H}_{2}$ is produced via the three-body reaction must be compensated by the destruction rate to bring the system to a chemical and thermal equilibrium, the abundances of which are related through the following well-known Saha equation:

$\frac{n_{\mathrm{H}_{2}}}{n_{\mathrm{H}}^{2}}=\frac{z_{\mathrm{H}_{2}}}{z_{\mathrm{H}}^{2}}\left(\frac{h^{2}}{\pi m_{\mathrm{H}} k T}\right)^{3 / 2} \exp \left(\frac{E_{\mathrm{diss}}}{k T}\right)$,

where $n_{\mathrm{H}_{2}}$ and $n_{\mathrm{H}}$ are the number densities of molecular and atomic hydrogen, respectively, $z_{\mathrm{H}_{2}}$ and $z_{\mathrm{H}}$ are the partition functions of molecular and atomic hydrogen, $E_{\text {diss }}$ is the dissociation energy of the hydrogen molecule, $k$ is the Boltzmann constant, $h$ is Planck's constant, and $T$ is the temperature. In LTE, the adopted values of the rate coefficient for the $\mathrm{H}_{2}$ collisional dissociation $\left(k_{\text {diss }}\right)$ must be consistent with the three-body formation rate coefficient $\left(k_{\text {form }}\right)$ in the sense that each pair of rate coefficients satisfies the chemical equilibrium condition $k_{\text {form }} / k_{\text {diss }}=$ $K$, where $K$ is the equilibrium constant.

Once the gas density reaches a value $\sim 10^{10} \mathrm{~cm}^{-3}$, the cloud becomes optically thick to the strongest of $\mathrm{H}_{2}$ lines. Using the Sobolev approximation (as described in Yoshida et al. 2006), the $\mathrm{H}_{2}$ cooling rate in this regime can be expressed as

$\Lambda_{\mathrm{H}_{2}, \text { thick }}=\sum_{u, l} h v_{u l} \beta_{\mathrm{esc}, \mathrm{ul}} A_{u l} n_{u}$,

where $n_{\mathrm{u}}$ is the number density of the hydrogen molecules in upper energy level $u ; A_{u l}$ is the spontaneous radiative transition rate, also known as Einstein coefficient, for a transition between $u$ and $l ; h v_{u l}$ is the energy difference between $u$ and $l$; and $\beta_{\text {esc }, u l}$ is the escape probability associated with this transition, i.e., the probability that the emitted photon can escape from the region of interest. In the high-density regime $\left(\sim 10^{14} \mathrm{~cm}^{-3}\right)$, the gas goes through a phase of cooling instability due to a strong increase in the cooling rate by $\mathrm{H}_{2}$ collisional induced emission (CIE; see, e.g., Ripamonti \& Abel 2004, for detailed discussion). Above the central density $\sim 10^{16} \mathrm{~cm}^{-3}$, the gas becomes completely optically thick to the continuum radiation (Yoshida et al. 2008). At this point the remaining $\mathrm{H}_{2}$ dissociates via reactions $(5,6)$, and hence cools the gas to collapse further. Once all the $\mathrm{H}_{2}$ dissociates, the gas becomes fully adiabatic with core mass $\sim 0.01 M_{\odot}$ surrounded by a massive, dense envelope that accretes matter rapidly (Clark et al. 2011a).

The vanguard numerical simulations (e.g., Abel et al. 2002; Yoshida et al. 2006; Bromm et al. 2009) propose the formation of massive (typically $\sim 20-50 M_{\odot}$ ) primordial protostars. This calculation result, however, contrasts with the present day star formation in which protostars with masses less than $1 M_{\odot}$ are formed (Kroupa 2002; Chabrier 2003). The recent improved and high resolution numerical simulations have inferred that the disk around the primordial core is unstable and fragments to form a small $\mathrm{N}$ system with low-mass stars, instead of a single protostar (e.g., Stacy et al. 2010; Clark et al. 2011; Hirano et al. 2014; Hartwig et al. 2015).

In the literature, there are appreciable indications that the collapsing cloud from which the protostar forms could have strong rotational support (Larson 1969, 1984). The cloud's rotation can affect the dynamical as well as the thermal evolution of gas and consequenty determine the ensuing properties of the Pop III stars (Bodenheimer 1995; Matsumoto et al. 1997). The consequences of the cloud's rotation on the chemical signature of the zero-metallicity primordial stars have been studied using stellar models (Meynet 2009, and references therein). With the use of the sink particle technique (discussed in Sect. 2) in the smoothed particle hydrodynamics ( $\mathrm{SPH}$ ) simulations, Stacy et al. (2011) have discussed the rotation velocity of the first stars, angular momentum transfer and the internal structure of the newborn protostars (see also Stacy et al. 2013, for the Arepo simulations). The central protostar rotates with a significant fraction of the Keplerian velocity. There is however scatter in the radial velocity, temperature, and accretion rate. In a recent study, Hirano et al. (2014) performed radiation hydrodynamical simulations to follow the evolution of 100 primordial protostars. Although their simulations were in $2 \mathrm{D}$, they nicely compared the angular momentum of the cloud with the Pop III accretion rate. More recently, simulations and analytic models have shown the formation of the massive primordial stars in rapidly rotating disks in the presence of turbulence and UV backgrounds (Latif et al. 2013, and references therein). However, the extent to which the thermal and dynamical evolution of gas depends on the initial degree of rotation of the cloud has never been systematically tested. In addition, there is so far no clear understanding of how the cloud's rotation can regulate the concurrent heating and cooling process during the collapse.

The dependence of the resultant fragmentation on the cloud's initial rotation, however, has been shown in detail in previous studies (e.g., Machida et al. 2008b; Saigo et al. 2008). After performing a number of idealized numerical experiments, these parameterized studies have concluded that the formation of either binary or multiple systems depend highly on the initial rotation of the cloud. Nevertheless, these studies could not point out the influence on the thermal evolution of the primordial gas during collapse because their calculations adopted the model of equation of state (EOSs). In this work, we candidly scrutinize the role of initial rotation of the collapsing gas on the heating and cooling process that controls the chemothermal evolution of gas inside minihalos. In addition, we perform rigorous calculations in both the idealized as well as more realistic cosmological minihalos to thoroughly analyze the evolution of gas particles and their physical properties. This unique approach thus enables us to investigate in detail the thermal, chemical, and dynamical evolution of the baryonic matter in a full 3D simulation of Pop III collapse to understand better the physical process and the resulting fragmentation behavior that occurs once the first object is formed. Finally, we address important issues, such as the relation of the 
physical property and accretion phenomenon of the protostars on the initial rotation of the collapsing core. We also discuss the possibility of survival of Pop III stars until today.

The paper is organized in the following manner. In Sect. 2 we describe the numerical setup of the simulations and the initial conditions. In Sect. 3 we briefly discuss the relevant physical concepts of the problem with an emphasis on the heating and cooling process that determines the temperature evolution. The details of the velocity structure are outlined in Sect. 4 . We discuss the accretion phenomenon comprehensively, followed by the implication of this study for the fragmentation of primordial gas in Sect. 5. The long-term evolution of protostars are contoured in Sect. 6. We summarize the main points and draw our conclusions in Sect. 7.

\section{Simulations}

In order to follow the gravitational collapse, one needs to ensure that the gas evolution in the simulations should not depend on the choice of minihalos. We therefore use two completely different numerical setups: minihalos from the cosmological simulations of Greif et al. (2011) obtained from the hydrodynamic moving mesh code Arepo (Springel 2010) and the artificial minihalos with an initially uniform density distribution of gas particles. In the following, we describe the initial condition and setup of the simulations.

To investigate the cosmological minihalos, we use snapshots at the epoch when the central number density is just below $10^{6} \mathrm{~cm}^{-3}$, the onset of the crucial three-body $\mathrm{H}_{2}$ formation reaction. The mesh-generating points of Arepo can be interpreted as the Lagrangian fluid particles, which is the basic characteristic of the Gadget-2 SPH code (Springel 2005). At this time, the minihalos contain masses of $1030 M_{\odot}$ and $1093 M_{\odot}$ with maximum central temperature of $469 \mathrm{~K}$ and $436 \mathrm{~K}$, respectively. The complete physical nature of these minihalos (e.g., number density $(n)$, initial rotation $\left(\beta_{0}\right)$, maximum and minimum temperature, etc.) is summarized in Table 1 . We use the Arepo output of these minihalos (MH-1 and MH-2 from Greif et al. 2011) as the initial conditions for our Gadget-2 implementation. Because of the conversion from the moving mesh to the SPH formalism (see, e.g., Smith et al. 2011; Dutta et al. 2015), we denote the minihalos as $\mathrm{CH} 1$ and $\mathrm{CH} 2$ for the Gadget-2 execution. The numerical resolution for $\mathrm{CH} 1$ and $\mathrm{CH} 2$ in SPH Gadget-2 simulations (for $100 \mathrm{SPH}$ particles) is $\sim 10^{-2} M_{\odot}$.

For the artificial setup, we use the initial conditions that permit us to carry out a set of methodical numerical experiments. The randomly distributed gas particles have been settled using the periodic boundary conditions in a box. During this initial phase, we keep the gas temperature fixed and do not follow the primordial chemical network. Once the gas particles are settled, we are in a position to perform the simulations for the collapse of gas due to self-gravity.

The gas particles are initially uniformly distributed in a spherical cloud of size $R_{0} \sim 2.7 \mathrm{pc}$ and a total mass of $M=$ $2982 M_{\odot}$. The number density is $n=10^{3} \mathrm{~cm}^{-3}$ and the temperature $T=200 \mathrm{~K}$. These initial conditions are equivalent to the primordial gas clumps that collapse (see, e.g., Abel et al. 2002; Yoshida et al. 2006). All clouds are modeled with 5 million SPH particles, and the mass of a single SPH particle is $m_{\mathrm{SPH}}=5.9 \times 10^{-4} M_{\odot}$. Therefore, the numerical resolution (roughly $100 \mathrm{SPH}$ particles) is $0.06 M_{\odot}$. The free-fall time for the uniform density distribution is $t_{\mathrm{ff}}=\sqrt{3 / 32 \pi G \rho}=1.37 \mathrm{Myr}$ and the sound crossing time is $t_{\mathrm{sc}} \approx 5 \mathrm{Myr}$. As the free-fall timescale
Table 1. Physical properties of the cosmological minihalos are summarized.

\begin{tabular}{lll}
\hline \hline $\begin{array}{l}\text { Halo } \\
\text { properties }\end{array}$ & $\mathrm{CH} 1$ & $\mathrm{CH} 2$ \\
\hline$n\left(\mathrm{~cm}^{-3}\right)$ & $10^{6}(\max ) 71(\min )$ & $10^{6}(\max ) 85(\min )$ \\
$T(\mathrm{~K})$ & $469(\max ) 59(\min )$ & $436(\max ) 54(\min )$ \\
mass $\left(M_{\odot}\right)$ & 1030 & 1093 \\
$n-\mathrm{SPH}$ & 690855 & 628773 \\
resolution $\left(M_{\odot}\right)$ & $1.3 \times 10^{-2}$ & $1.4 \times 10^{-2}$ \\
$\beta_{0}$ & $0.035(\max ) 0.025(\min )$ & $0.042(\max ) 0.03(\min )$ \\
\hline
\end{tabular}

Notes. $n$ denotes the number density, $T$ the temperature, $n$-SPH the number of SPH particles and $\beta_{0}$ the rotation parameter, respectively. The numerical resolution is calculated for the $100 \mathrm{SPH}$ particles.

is shorter than the sound-crossing timescale, the clouds immediately start to collapse under their self-gravity. The clouds are then given different degrees of solid body rotation and are not subject to the internal turbulent motions. The strength of rotational support can be described by the $\beta_{0}$-parameter (Sterzik et al. 2003),

$\beta_{0}=\frac{E_{\mathrm{rot}}}{E_{\mathrm{grav}}}=\frac{R_{0}^{3} \Omega^{2}}{3 G M}$,

where $\Omega$ is the angular velocity, and $E_{\text {rot }}$ and $E_{\text {grav }}$ are the magnitudes of the rotational and the gravitational energies, respectively. We perform ten different numerical experiments with $\beta_{0}=0.0,0.005,0.007,0.01,0.02,0.04,0.05,0.07,0.1,0.2$.

As the collapse progresses in the central region of the cloud, it is extremely difficult to simulate the higher density regime because of shorter timescale. To overcome this problem, we use the sink particle technique in which the high-density region is replaced by a single sink particle with appropriate boundary conditions (see, e.g., Bate et al. 1995; Krumholz et al. 2004; Jappsen et al. 2005). The sink particle can then be assumed to be (or at least approximated by) a growing protostar. The density threshold for the sink particles to form is set as the number density of $5 \times 10^{13} \mathrm{~cm}^{-3}$, at which point the gas has a temperature of $\sim 1000 \mathrm{~K}$. The sink particle can accrete gas particles within its accretion radius $r_{\mathrm{acc}}$, which we fix at $6 \mathrm{AU}$, the Jeans radius at the density threshold for sink creation. The corresponding Jeans mass for both the cosmological as well as the artificial clumps is $0.06 M_{\odot}$, so we can resolve both the cosmological and artificial minihalos. The softening parameter of the sinks is 1.2 AU. In order to avoid spurious formation of new sink particles out of the gas, the sink particles is prevented further from forming within $2 r_{\text {acc }}$ of one another.

We follow Clark et al. (2011a) for the implementation of the external pressure term and a time-dependent primordial chemical network. To model a constant pressure boundary, we used a modified version of the Gadget 2 momentum equation,

$\frac{\mathrm{d} v_{i}}{\mathrm{~d} t}=-\sum_{j} m_{j}\left[f_{i} \frac{P_{i}}{\rho_{i}^{2}} \nabla_{i} W_{i j}\left(h_{i}\right)+f_{j} \frac{P_{j}}{\rho_{j}^{2}} \nabla_{i} W_{i j}\left(h_{j}\right)\right]$,

where the contribution from the external pressure $\left(P_{\text {ext }}\right)$ is subtracted from both $P_{i}$ and $P_{j}$ (i.e., $P_{i}$ and $P_{j}$ are replaced by $P_{i}-P_{\text {ext }}$ and $P_{j}-P_{\text {ext }}$, respectively). All quantities have their usual meaning. The chemical network includes primordial hydrogen, helium, and deuterium to model the chemical and thermal evolution of the metal-free gas inside minihalos. The details of all the chemical reactions are given in Glover \& Abel (2008) 

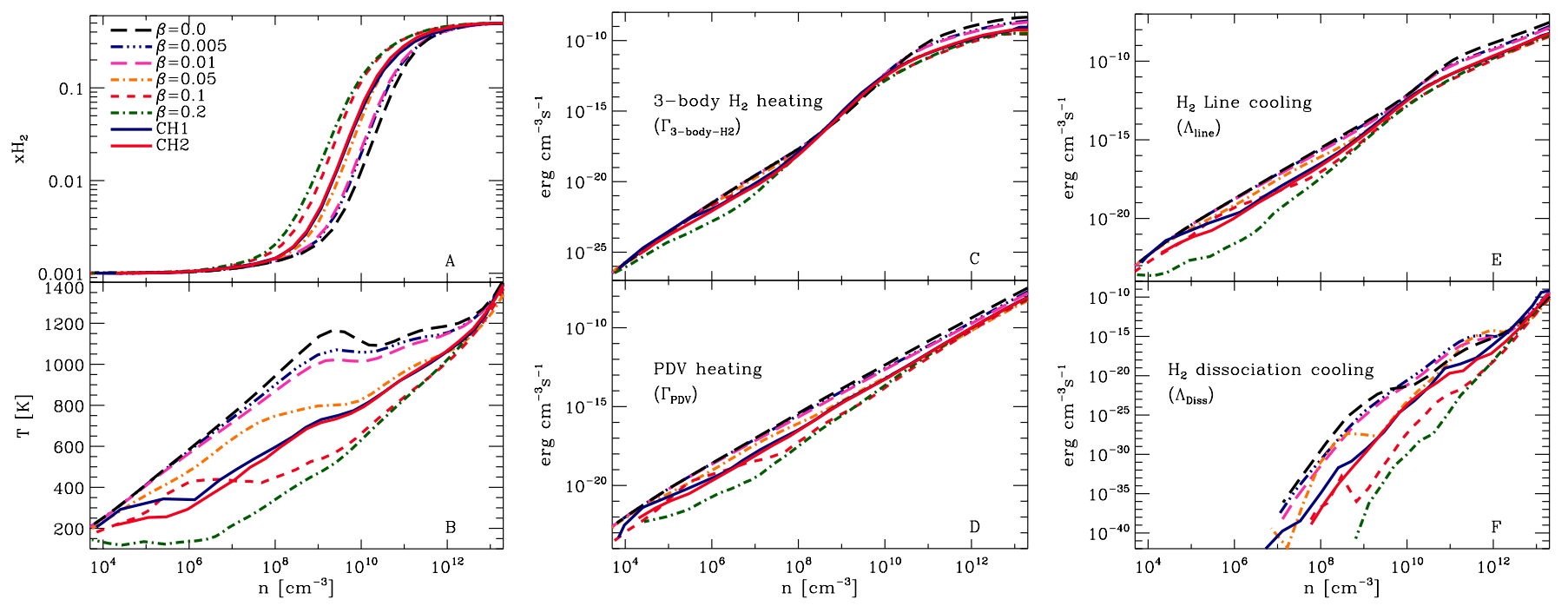

Fig. 1. Radial logarithmic binned, mass-weighted averages of the $\mathrm{H}_{2}$ fraction $\mathbf{A}$ ), temperature B), and various heating and cooling rates (C) to $\mathbf{F}$ )) are plotted as a function of density for different degrees of initial rotation $\beta_{0}$, just before the formation of the first sink.

and references therein. We adopt the intermediate three-body $\mathrm{H}_{2}$ rate coefficient $7.7 \times 10^{-31} T^{-0.464} \mathrm{~cm}^{6} \mathrm{~s}^{-1}$ proposed by Glover (2008).

\section{Heating and cooling rate}

In this section, we investigate the cooling and heating mechanism associated with the emission, chemical reactions and gas contraction during the collapse of the cloud under its own gravity. The differences in the cooling and heating rates can force the gas to choose different paths for its thermodynamic evolution.

If we assume that the gas density $(\rho)$ evolves with the freefall time $\left(t_{\mathrm{ff}}\right)$ of the gas, i.e., $\mathrm{d} \rho / \mathrm{d} t=\rho / t_{\mathrm{ff}}$ (see, e.g., Omukai 2000; Glover \& Savin 2009), the thermal evolution can then be followed by solving the energy equation

$\frac{\mathrm{d} \epsilon}{\mathrm{d} t}=\frac{p}{\rho} \frac{\mathrm{d} \rho}{\mathrm{d} t}-\Lambda+\Gamma$,

where $\epsilon$ is the energy per unit volume in the gas, and $\Lambda$ and $\Gamma$ are the cooling and heating rates, respectively, in units of erg $\mathrm{s}^{-1} \mathrm{~cm}^{-3}$. Figure 1 shows the physical conditions in the gas once the central region has collapsed to a density of $\sim 5 \times 10^{13} \mathrm{~cm}^{-3}$, i.e., just before the first sink formation. The panels show mass-weighted averages of the properties of individual SPH particles within the radial logarithmic bins.

The three-body $\mathrm{H}_{2}$ formation heating rate provides chemical heating associated with the release of $4.48 \mathrm{eV}$ each time a $\mathrm{H}_{2}$ molecule forms. Because the $\mathrm{H}_{2}$-fractions are almost similar (Fig. 1A), there is no consequential effect of the three-body $\mathrm{H}_{2}$ heating on the cloud's initial rotation (Fig. 1C). However, we find that there are substantial temperature differences between the clouds (Fig. 1B). For example, the temperature of the cloud with $\beta=0.2$ is almost $40-60 \%$ lower than that with $\beta_{0}=0.005$. This is because a higher degree of the rotational support slows down the contraction and reduces the amount of compressional heating (Fig. 1D). Thus, the cloud with $\beta=0.2$ has a temperature of roughly $T \leq 200 \mathrm{~K}$ due to a slower collapse and, hence, less efficient compressional heating, whereas the temperature is nearly $1100 \mathrm{~K}$ in case of $\beta=0.0$ or $\beta=0.005$.

The rapid conversion of atomic to molecular hydrogen during the three-body reaction cools the gas less than the free-fall time and hence causes chemothermal instability (Dutta 2015a). At high densities the heating rate is as high as the line-cooling rate (Fig. 1E), however, again with slight differences over the range of $\beta_{0}$ modeled. At equilibrium, the formation rate is balanced by the dissociation, and hence the dissociation cooling rate behaves in the same way with density as the heating rate. The dissociation cooling rate varies between clouds, with that for $\beta_{0}=0.005$ nearly 10 orders of magnitude higher than for the $\beta_{0}=0.2$ model (Fig. $1 \mathrm{~F}$ ) in the density range where the three-body reaction dominates. We therefore conclude that it is indeed the compressional heating $(p \mathrm{~d} V)$ that determines the thermal evolution of gas, which strongly depends on the initial degree of rotation $\left(\beta_{0}\right)$.

At this point, it is worth pointing out that the temperature evolution of the cosmological minihalos are similar to that of the idealized cases. As expected, both $\mathrm{CH} 1$ (with $\beta_{0} \approx 0.035$ ) and $\mathrm{CH} 2$ (with $\beta_{0} \approx 0.042$ ) have the temperature variation that falls in between the highest and lowest $\beta_{0}$ modeled throughout the density space. This also confirms that our varied parameter study with idealized clumps actually represents the cosmological initial conditions of the minihalos.

\section{Velocity structure}

In this section, we study the dynamics of the gas particles that can arise as a result of the chemical and thermal evolution of the clouds. We therefore take a closer look at the velocity of the gas associated with the cloud collapse. The radial profiles of the gas show the mass-weighted averages within logarithmic bins and are taken just before the formation of the first sink.

We find that there are considerable differences in radial velocities between the clouds (Fig. 2A). The radial velocity of the cloud with $\beta_{0}=0.005$ is almost $30-40 \%$ higher than the cloud with $\beta_{0}=0.2$. This is consistent with the fact that a lower rate of compressional heating for the gas of the collapsing core implies a lower radial velocity, which is nearly comparable with the sound speed (Fig. 2C).

In order to quantify the degree of rotational support, we plot the rotational velocity (Fig. 2B) and the ratio of the rotational velocity $\left(v_{\mathrm{rot}}\right)$ to the Keplerian velocity $\left(v_{\mathrm{Kep}}\right)$, defined as $v_{\mathrm{K}}=\sqrt{G M_{\mathrm{enc}}(r) / r}$, where $M_{\mathrm{enc}}(r)$ is the mass enclosed within the radius $r$ (Fig. 2F). We find that the rotational speed for all 

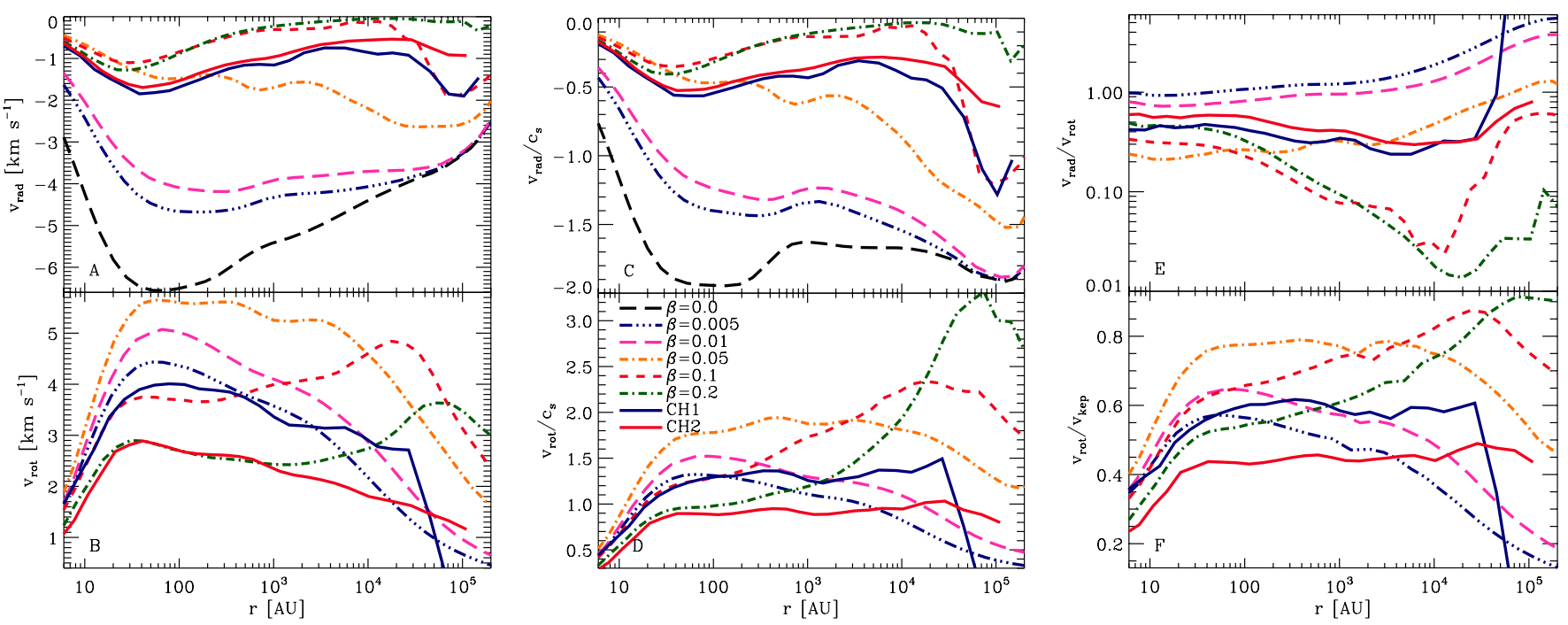

Fig. 2. Radial logarithmic binned, mass-weighted averages of the radial velocity A), rotational velocity B), radial velocity over sound speed C), rotational velocity over sound speed D), rotational velocity over Keplerian speed $\mathbf{E}$ ), and radial velocity over rotational velocity are plotted as a function of radius, just before the first protostar forms. The initial strength of rotation introduces a scatter in the velocity.

$\beta_{0}$ modeled is well below that required for rotational support by a factor of 5 to 7 . This is consistent with previous cosmological simulations (e.g., Yoshida et al. 2008; Turk et al. 2009), which argued that the collapsing gas cloud has the ratio $v_{\text {rot }} / v_{\text {Kep }} \sim 0.5$.

For the $\beta_{0}=0.2$ case, the rotation speed varies between 0.3 and 0.9 times the Keplerian velocity, indicating that this cloud has gone through an efficient phase of angular momentum redistribution (Dutta 2015b). However, for $\beta_{0}>0.05$ the collapsing cloud is almost completely rotationally supported throughout and the gas at higher densities is relatively cold. All clouds are consequently sub-Keplerian, and the radial velocities for all clouds are comparable to the rotational velocities within $100 \mathrm{AU}$ (Fig. 2E). From this discussion, we can infer that the cloud with higher rotation transfers the angular momentum more efficiently and hence becomes Keplerian in the outer regime of the collapsing core (for instance, $v_{\text {rot }} \sim v_{\text {Kep }}$ for $r \geq 10^{4}$ AU for $\beta_{0}=0.2$ ). Thus the outer regime, which is likely to form a Keplerian disk due to rotation, becomes unstable enough from accreting mass and, consequently, has higher chance to fragment (as we see in the next section). We conclude that the cloud's initial rotation plays a pivotal role in the dynamical evolution of the gas by affecting the amount of rotational support even at later stages of the collapse.

\section{Mass accretion and fragmentation}

During collapse, the angular momentum is transported to smaller scales, resulting in the formation of rotationally supported disklike structures (Stacy et al. 2011; Greif et al. 2012). However, the rotation is not sufficient to hold the collapse of the disk, which then fragments into multiple objects (Clark et al. 2011; Dutta et al. 2015). We thus gauge the accretion and gas instability carefully, as predicted by the properties of the gas, to check whether there is any hint of fragmentation already present before the formation of the first sink.

We follow Abel et al. (2002) to study the mass accretion rate, estimated as $\dot{M}(r)=4 \pi r^{2} \rho(r) v_{\text {rad }}(r)$, as a function of radius for different degrees of initial rotation (Fig. 3A). We also define the accretion time, $t_{\mathrm{acc}}=M_{\mathrm{enc}}(r) / 4 \pi \rho v_{\mathrm{rad}} r^{2}$. For all simulations, $\dot{M}$ has a maximum at $\sim 20-40$ AU. Given that $\mathrm{H}_{2}$ line cooling becomes optically thick at the corresponding densities, $\dot{M}$ for all simulations converges in this range, as the gas looses its ability to cool efficiently. We would, however, like to point out that the scale, where $\mathrm{d} M / \mathrm{d} t$ becomes maximum, may slightly change with the choice of density threshold for the sink formation. For example, if we choose $n \sim 10^{15} \mathrm{~cm}^{-3}$ for a sink particle to form, the collapse of the inner structure proceeds and hence the mass accretion rate reaches a maximum at $\sim 15-30 \mathrm{AU}$, depending on the initial degree of rotation. However, this is not a substantial issue compared to the overall thermal nature of the collapsing cloud. For slowly rotating clouds, the accretion rate is substantially higher $\left(\sim 0.1 \mathrm{yr}^{-1}\right)$ and the accretion time is on the order of free-fall time (Fig. 3C).

Since the collapsing core becomes Jeans unstable by accreting more and more mass, we check the strength of the gravitational instability by measuring the number of the BonnorEbert masses $\left(M_{\mathrm{BE}}\right)$ contained in the central dense volume (Ebert 1955; Bonnor 1956). We compute the Bonnor-Ebert masses as $M_{\mathrm{BE}}=1.18\left(c_{\mathrm{s}}^{4} / G^{3 / 2}\right) P_{\mathrm{ext}}^{-1 / 2} \approx 20 M_{\odot} T^{3 / 2} n^{-1 / 2} \mu^{-2} \gamma^{2}$ (Abel et al. 2002), where $c_{\mathrm{s}}$ is the sound speed, $P_{\text {ext }}$ is the external pressure that we assume to be equal to the local gas pressure, $\mu$ is the mean mass per particle, and $\gamma=5 / 3$ is the adiabatic index, respectively. Within the central $10^{4} \mathrm{AU}$ regime, the enclosed gas mass for all values of $\beta_{0}$ contains a roughly equal number of Bonnor-Ebert masses, although with a factor of two between the highest and lowest values of $\beta_{0}$ (Fig. 3B). However, in the outer regime (i.e., $r \geq 10^{4} \mathrm{AU}$ ), the clouds with $\beta_{0}=0.1$ and $\beta_{0}=0.2$ contain roughly 7 and 10 Bonnor-Ebert masses respectively. This is because the higher rotating clouds become close to Keplerian in the outer regime (as seen in the previous section) and tend to form a disk-like structure. As the higher rotation obstructs the infalling gas particles, the disk gradually accumulates enough Jeans masses through accretion and becomes gravitationally unstable to fragmentation.

Alongside the Bonnor-Ebert analysis, we also compare all relevant timescales associated with the cloud collapse. Figure 3C represents the accretion timescale $\left(t_{\text {acc }}\right)$ over free-fall timescale $\left(t_{\mathrm{ff}}\right)$. For a higher rotating cloud, the accretion time is much longer than the free-fall time. This is consistent with the above discussion and an alternate way to explain the mass accretion features of clouds with different $\beta_{0}$-models shown in Fig. $3 \mathrm{~A}$. 


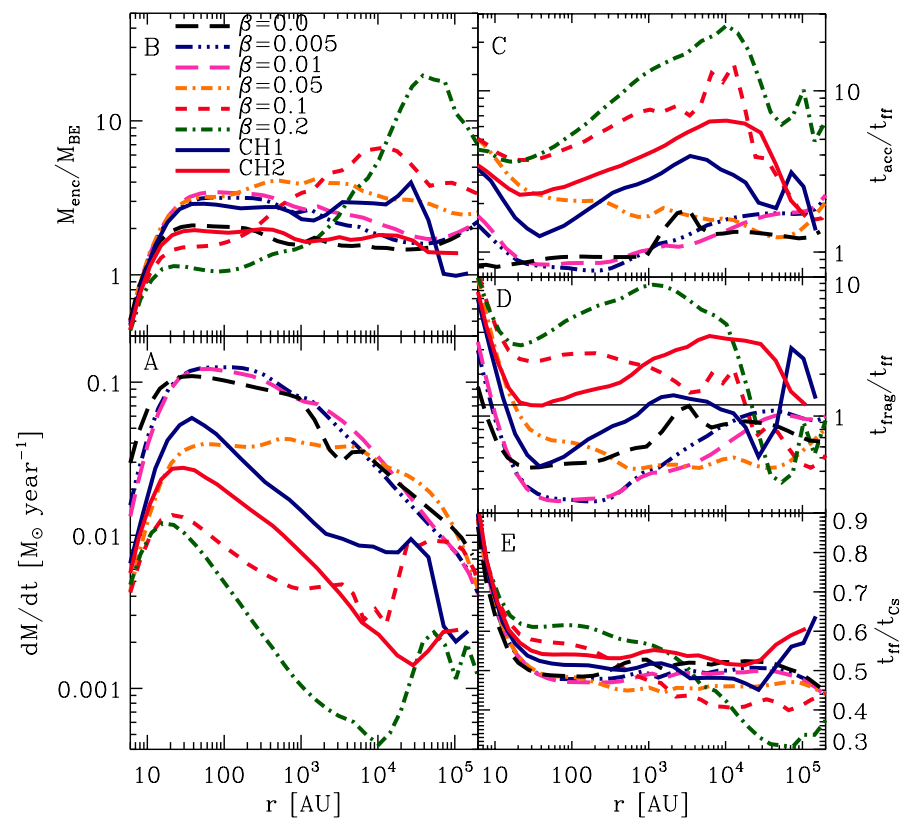

Fig. 3. Radial logarithmic binned, mass-weighted averages of the mass accretion rate $\mathbf{A}$ ), the number of Bonnor-Ebert masses $\mathbf{B}$ ), the accretion time over free-fall time $\mathbf{C}$ ), the fragmentation time over free-fall time D), and the free-fall time over sound crossing time E) are plotted as a function of radius, just before the formation of the first sink.

In Fig. 3D, we consider the fragmentation timescale, defined as $t_{\text {frag }} \equiv M_{\mathrm{BE}} / \dot{M}$ (e.g., Dopcke et al. 2013), and compare it with the free-fall timescale. We know that fragmentation generally occurs when the dynamical timescale of the central collapse becomes larger than the collapse timescale of individual density fluctuations. Here the free-fall timescale represents the dynamical timescale. In the outer regime $\left(r \geq 2 \times 10^{4} \mathrm{AU}\right)$, which is likely to form a disk, the fragmentation time becomes shorter than the free-fall time, especially for higher rotating clouds. We conclude here that the clouds with larger $\beta_{0}$ collapse slowly from high rotation and tend to form a disk-like structure that becomes Keplerian and gravitationally unstable by accreting mass, heralding fragmentation.

\section{Protostellar system}

The study by Machida et al. (2008b) shows that any fragmentation only takes place after the disk formation, and that clouds with higher $\beta_{0}$ tend to fragment at lower densities. We examine how the circumstellar accretion disk that formed in the idealized, as well as more realistic cosmological minihalos, becomes locally unstable and evolves for different degrees of initial rotation.

Figure 4 shows the column density and column-weighted temperature distribution in the inner $2000 \mathrm{AU}$ at the end of the simulations. These images clearly reflect that all simulations fragment to form a small $N$-body system, comprised of secondary protostars, within the time in which $\sim 30 M_{\odot}$ of material are accreted onto the sink particles. It is not surprising that clouds with a higher level of rotational support exhibit the disklike structure on several scales within the central region, whereas slowly rotating clouds are more likely to be centrally condensed.

The top left panel of Fig. 5 shows the time evolution of the total mass accreted onto all the sinks $\left(\Sigma M_{*}\right)$ and the maximum sink mass $\left(M_{* \max }\right)$ in the period over which the sink formation occurs. We find that the cloud with zero rotation attains the $30 M_{\odot}$ within a few hundred years after the formation of the first sink particle, whereas rotationally supported clouds take $100-1000 \mathrm{yr}$, depending on the initial strength of rotation. Figure 5 also shows the time evolution of the total mass accretion rate $\left(\mathrm{d} M_{*} / \mathrm{d} t\right)$ onto all the sinks (same as Fig. 3A) for different values of $\beta_{0}$. As expected, the mass accretion rate is larger for the slowly rotating clouds. In each case, $\mathrm{d} M_{*} / \mathrm{d} t \mathrm{de}$ creases with time until further sink particles form, and then the total accretion rate increases again, however, this time with large temporal variations. The mass accretion rate for the idealized cloud with $\beta_{0}=0.04$ is similar to that of the cosmological minihalo $\mathrm{CH} 2$, which has a rotation parameter $\sim \beta_{0}=0.042$. This again confirms that our idealized clumps with varying $\beta_{0}$ can actually be the representatives of cosmological initial conditions to investigate the thermodynamical evolution of gas and the resulting fragmentation behavior of the sinks.

Figure 6A shows the number of sinks for different values of $\beta_{0}$, with rotationally supported clouds fragmenting the most. This is consistent with the recent study by Becerra et al. (2015), who have used Arepo simulations to investigate the dependence of the high accretion rate and efficient cooling of the gas on the fragmentation of the disk. However, Fig. 6A shows some scatter in the numbers, indicating the presence of statistical fluctuations that can be removed by pursuing more realizations to achieve a desired degree of accuracy. Figure $6 \mathrm{~B}$ shows the time taken for all the clouds to accumulate $\sim 30 M_{\odot}$. As expected, higher rotating clouds take longer compared to their slowly rotating counterparts. Figure 6C shows the distance at which the primordial protostars form from the center of the cloud. The red line represents the mean distances of all protostars $\left(R_{\text {dist }}\right)$, which follows a power-law relationship with the cloud's initial rotation, $R_{\text {dist }} \propto \beta_{0}^{3 / 4}$. The protostars of the slowly rotating clouds form near the center $(\leq 300 \mathrm{AU})$, while the others spread over larger distances of $5000 \mathrm{AU}$, as the conservation of angular momentum acts to move protostars to larger radii.

Another trend we find in our simulations is that a number of protostars are ejected from the central gas cloud, as seen in cosmological simulations (Greif et al. 2011). Although the resolution used in those studies was higher than our resolution, we still find ejection from the cluster. Figure 7 shows the radial velocity and the ratio of the radial velocity to the escape velocity for all protostars. The position of the sinks is measured from the center of mass of all the sinks. The escape velocity of the sinks is defined as $v_{\mathrm{esc}}=\sqrt{2 G M_{\mathrm{enc}}(r) / r}$, where $M_{\mathrm{enc}}(r)$ is the total mass (gas + sinks) that is enclosed within the radius $r$. The radial velocities of the sinks formed with lower $\beta_{0}$ are below that required to be kicked out of the cluster. They tend to remain within the cluster and continue to accrete. For faster-rotating clouds, some protostars move from the cluster with radial velocities exceeding the escape velocity. There is, therefore, a greater chance that some protostars will be ejected, opening up the possibility that they could survive until the present day.

At this point we would like to point out that we have not mentioned the behavior of sinks for the $\beta_{0}=0.2$ case. The thermodynamical evolution of this cloud is considerably different from other cases, so it is important to compare the fragmentation behavior of $\beta_{0}=0.2$ cloud with others. However, because of very fast rotation it has been extremely tough to run the simulation up to the point where the total mass of all sinks reaches $\sim 30 M_{\odot}$. The simulation with $\beta_{0}=0.2$ stops much before compared to other simulations. For instance, simulation with $\beta_{0}=0.2$ stops when the total mass of sinks is only around 3-4 $M_{\odot}$ (and to reach up to that epoch of time; it takes around one month to run on a supercomputer that is based on graphical processing units, HPC-GPU Cluster Kolob). This is usually 


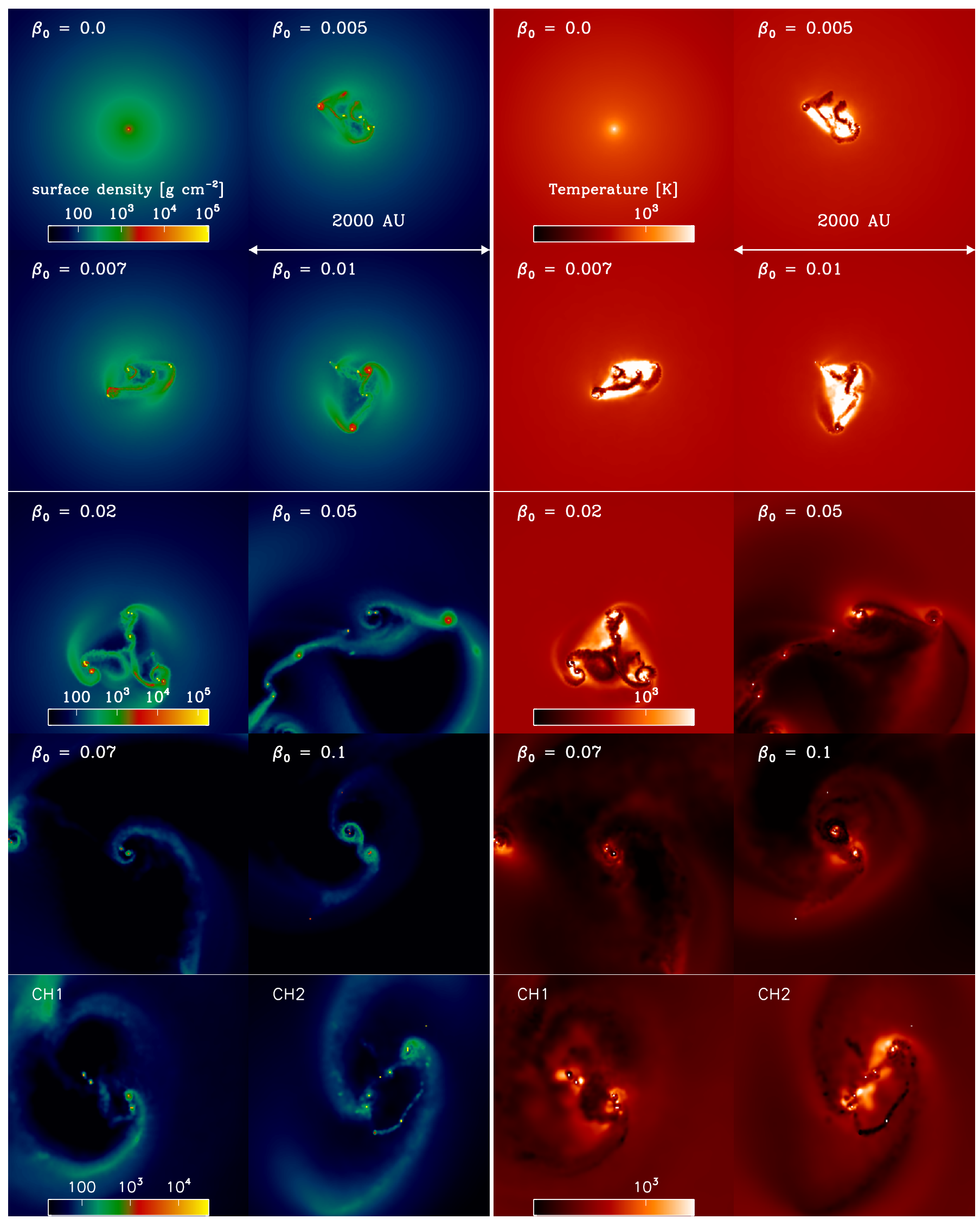

Fig. 4. Column density and column-weighted temperature distribution in a region of 2000 AU centered around the first protostar for different strengths of the initial rotation of the cloud are shown when a total of $\sim 30 M_{\odot}$ have been converted into, or accreted onto, sink particles. 

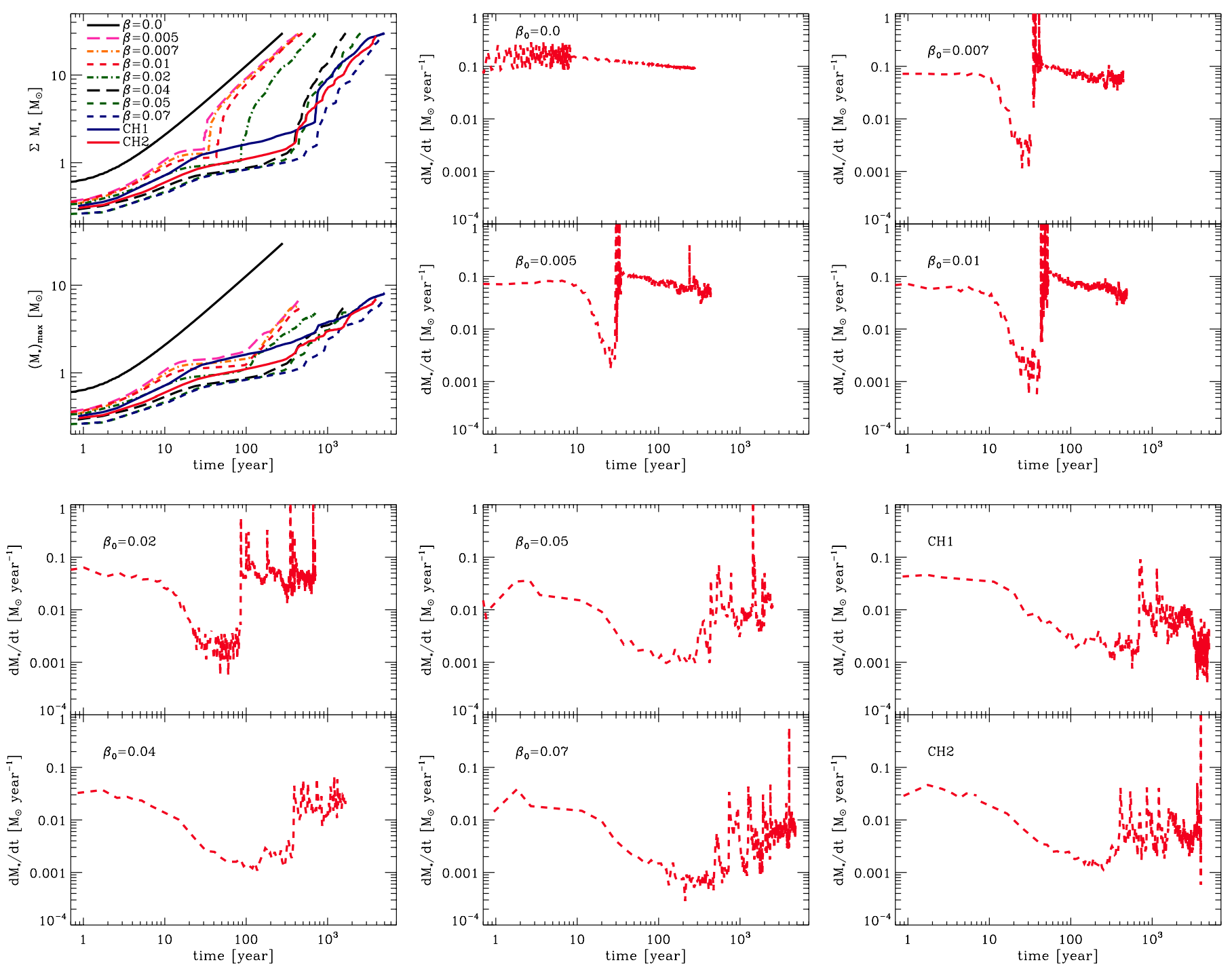

Fig. 5. Time evolution of the protostellar system: total mass of all the sink particles and the most massive sink particle are plotted as a function of time (top left). Total mass accretion rate by all sink particles are shown as a function of time (for others rotation parameter $\beta_{0}$; same as Fig. 3A). The cloud with zero rotation attains $30 M_{\odot}$ within a few hundred years after the formation of the first sink particle. The rotationally supported cloud takes $\sim 100-1000 \mathrm{yr}$, depending on the initial strength of rotation $\left(\beta_{0}\right)$. The mass accretion rate decreases with time until further sink particles form.

expected as the fast rotation can impede the collapse. We find a limiting value of $\beta_{0}=0.1$ for the simulation to run up to the epoch when the mass of sinks attains a value of $30 M_{\odot}$. However, Figs. 4-7 still allow us to extrapolate and predict the expected results.

\section{Summary and discussion}

We have minutely investigated the dependence of the thermodynamical evolution of primordial star-forming gas on the initial degree of rotation of the cloud, and analyzed its influence on the resulting fragmentation of the circumstellar accretion disk. For this purpose, we performed a set of three-dimensional hydrodynamical simulations of Pop III gas collapse to pursue a systematic parameter study, specified by $\beta_{0}$, which spans two orders of magnitude for the amount of initial rotation, including two simulations runs with realistic cosmological initial conditions.

The cloud's initial strength of rotation introduces a significant impact on the intricate combinations of the heating and cooling process, leading to a scatter in the temperature evolution of the collapsing gas. Clouds with slower rotation collapse faster and get heated as a result of the compressional heating. We also find that the dynamical evolution of the gas strongly depends on the initial strength rotation. Clouds with higher rotation form a Keplerian disk that becomes gravitationally unstable by accreting the infalling mass. Therefore, any change in the thermodynamical evolution introduces substantial difference in the number of Jeans mass, which determines the susceptibility to the fragmentation of the gas between the clouds with highest and lowest initial rotation.

In summary, a higher degree of rotation can hinder the infall, lead to a cooler gas, and result in more fragmentation. In addition, we find that the protostars with higher rotational support have larger spiral arms with lower accretion rates. We also point out that the newborn protostars are distributed in such a way to conserve the angular momentum, and some of them could have survived until today if they were of sufficiently low mass.

We conclude that the initial conditions of the primordial gas in the minihalos should be chosen scrupulously so as to simulate the long-term evolution and final fate of the primordial stars. 


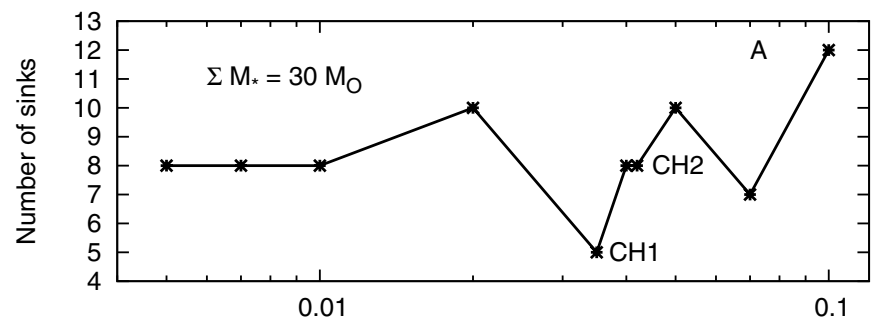

$\beta_{0}$
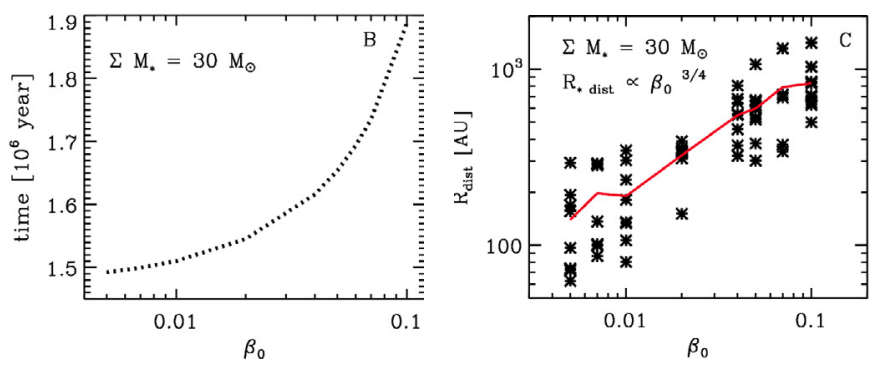

Fig. 6. Fragmentation behavior plotted for different degrees of initial rotation at the epoch when stellar system accretes $\sim 30 M_{\odot}$. The number of sinks A), time taken to accrete $\left.\sim 30 M_{\odot} \mathbf{B}\right)$ and the position of the sinks $\left(R_{\text {dist }}\right)$ from the center of mass is shown in $\mathbf{C}$ ) as a function of the rotation parameter $\beta_{0}$.
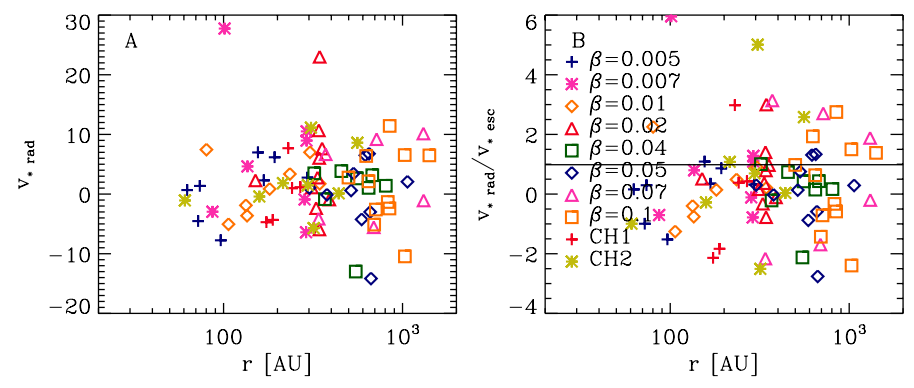

Fig. 7. Radial velocity $\mathbf{A}$ ) and the ratio of the radial velocity to the escape velocity B) of sink particles for different values of $\beta_{0}$ are plotted as a function of radius, at the epoch when the stellar system has accreted $\approx 30 M_{\odot}$. Some protostars with higher $\beta_{0}$ move away from the cluster, with the radial velocity exceeding the escape velocity.

Despite considerable computational efforts involved, we emphasize that we cannot accurately predict the final mass of the primordial protostars. We have neglected the effect of the magnetic fields, which can be important in minihalos (Machida et al. 2008a; Sur et al. 2010; Schleicher et al. 2010). Recent cosmological simulations show the importance of the amplification of even small seed fields (Federrath et al. 2011; Schober et al. 2012). In addition, the radiative feedback can significantly affect the thermal as well as chemical evolution of the gas (Wise \& Abel 2008; Whalen et al. 2010; Hosokawa et al. 2011), which is not included in our simulations. Notwithstanding, our approach to the problem enables us to provide good estimates for the overall trend of the accretion rate, thermodynamical evolution, and fragmentation behavior of gas in the rotating clouds in which we are particularly interested. Recent radiation hydrodynamic simulations (Hirano \& Yoshida 2013; Greif 2014) demonstrated the effect of UV radiative feedback on the mass accretion, thus constraining the mass spectrum of the first stars (Susa 2013). Moreover, there is still a discrepancy regarding the threebody $\mathrm{H}_{2}$ rate coefficient (Turk et al. 2011; Bovino et al. 2014; Dutta et al. 2015). However, the recent study by Forrey (2013) provides the currently best available rate that is in good agreement with Palla et al. (1983) at high temperatures and Abel et al. (2002) at lower temperatures. It is therefore of strategic interest to accurately simulate the formation of the first stars in the Universe with the best available rates, inclusion of the magnetic fields, and radiative feedback in the next-generation avant-garde SPH codes.

Acknowledgements. The author wishes to thank Prateek Sharma, Biman Nath, David Sobral, and Dominik Schleicher for thoroughly checking the manuscript and for enormous constructive suggestions. The author also acknowledges Kazu Omukai, Athena Stacy, and the referee for helpful and worthwhile comments. The present work is supported by the Indian Space Research Organization (ISRO) grant (No. ISRO/RES/2/367/10-11) and Department of Science and Technology (DST) grant (Sr/S2/HEP-048/2012). The author is grateful to the Centre for Theoretical Studies (CTS) at the Indian Institutes of Technology Kharagpur and Raman Research Institute for the financial support and hospitality. The author would also like to thank the Department of Physics, Indian Institute of Technology (Banaras Hindu University) at Varanasi and the InterUniversity Center for Astronomy and Astrophysics (IUCAA) at Pune for local hospitality.

\section{References}

Abel, T., Bryan, G. L., \& Norman, M. L. 2000, ApJ, 540, 39 Abel, T., Bryan, G. L., \& Norman, M. L. 2002, Science, 295, 93 Barkana, R., \& Loeb, A. 2001, Phys. Rep., 349, 125

Bate, M. R., Bonnell, I. A., \& Price, N. M. 1995, MNRAS, 277, 362

Becerra, F., Greif, T. H., Springel, V., \& Hernquist, L. E. 2015, MNRAS, 446 2380

Bodenheimer, P. 1995, ARA\&A, 33, 199

Bonnor, W. B. 1956, MNRAS, 116, 351

Bovino, S., Schleicher, D. R. G., \& Grassi, T. 2014, A\&A, 561, A13

Bromm, V., \& Larson, R. B. 2004, ARA\&A, 42, 79

Bromm, V., \& Yoshida, N. 2011, ARA\&A, 49, 373

Bromm, V., Yoshida, N., Hernquist, L., \& McKee, C. F. 2009, Nature, 459, 49

Clark, P. C., Glover, S. C. O., Klessen, R. S., \& Bromm, V. 2011a, ApJ, 727, 110

Clark, P. C., Glover, S. C. O., Smith, R. J., et al. 2011b, Science, 331, 1040

Chabrier, G. 2003, PASP, 115, 763

Ciardi, B., \& Ferrara A. 2005, Space Sci. Rev., 116, 625

Couchman, H. M. P., \& Rees, M. J. 1986, MNRAS, 221, 53

Dopcke, G., Glover, S. C. O., Clark, P. C., \& Klessen, R. S. 2013, ApJ, 766, 103

Dutta, J. 2015a, ApJ, 811, 98

Dutta, J. 2015b, Astrophys. Space Sci., accepted [arXiv: 1512.01656]

Dutta, J, Nath, B. B., Clark, P. C., \& Klessen, R. S. 2015, MNRAS, 450, 202

Ebert, R. Z. 1955, Astrophysics, 37, 217

Federrath, C., Sur, S., Schleicher, D. R. G., Banerjee, R., \& Klessen, R. S. 2011 , ApJ, 731, 62

Forrey, R. C. 2013, ApJ, 773, L25

Glover, S. 2013, Astrophys. Space Sci. Libr., 396, 103

Glover, S. C. O. 2008, in First Stars III, Chemistry and Cooling in Metal-Free and Metal-Poor Gas, eds. B. O'Shea, \& A. Heger (New York: AIP), 990, 25 Glover, S. C. O., \& Abel, T. 2008, MNRAS, 388, 1627

Glover, S. C. O., \& Savin, D. W. 2009, MNRAS, 393, 911

Greif, T. H. 2014, MNRAS, 444, 1566

Greif, T. H., Springel, V., White, S. D. M., et al. 2011, ApJ, 737, 75

Greif, T. H., Bromm, V, Clark, P. C., et al. 2012, MNRAS, 424, 399

Haiman, Z., Thoul, A. A., \& Loeb, A. 1996, ApJ, 464, 523

Hartwig, T., Clark, P. C., Glover, S. C. O., Klessen, R. S., \& Sasaki, M. 2015, ApJ, 799, 114

Hirano, S., \& Yoshida, N. 2013, ApJ, 763, 52

Hirano, S., Hosokawa, T., Yoshida, N., et al. 2014, ApJ, 781, 60

Hosokawa, T., Omukai, K., Yoshida, N., \& Yorke, H. W. 2011, Science, 334, 1250

Jappsen, A. K., Klessen, R. S., Larson, R. B., Li, Y., \& Mac Low, M. M. 2005 A\&A, 435, 611

Kroupa, P. 2002, Science, 295, 82

Krumholz, M. R., McKee, C. F., \& Klein, R. I. 2004, ApJ, 611, 399

Larson, R. B. 1969, MNRAS, 145, L271

Larson, R. B. 1984, MNRAS, 206, 197

Latif, M. A., Schleicher, D. R. G., Schmidt, W., \& Niemeyer, J. 2013, ApJ, 772, L3

Loeb, A. 2010, How Did the First Stars and Galaxies Form? (Princeton: Princeton Univ. Press) 
Machida, M. N., Matsumoto, T., \& Inutsuka, S. 2008a, ApJ, 685 Machida, M. N., Omukai, K., Matsumoto, T., \& Inutsuka, S. I. 2008b, ApJ, 677, 813

Matsumoto, T., Hanawa, T., \& Nakamura, F. 1997, ApJ, 478, 569

McDowell, M. R. C. 1961, Observatory, 81, 240

Meynet, G. 2009, Lect. Notes Phys., 765, 139

Omukai, K. 2000, ApJ, 534, 809

Omukai, K., \& Nishi, R. 1998, ApJ, 508, 141

O'Shea, B. W., \& Norman, M. L. 2006, ApJ, 648, 31

Palla, F., Salpeter, E. E., \& Stahler, S. W. 1983, ApJ, 271, 632

Peebles, P. J. E., \& Dicke, R. H. 1968, ApJ, 154, 891

Ripamonti, E., \& Abel, T. 2004, MNRAS, 348, 1019

Ritter, J. S., Safranek-Shrader, C., Gnat, O., Milosavljevi, M., \& Bromm, V. 2012, ApJ, 761, 56

Saigo, K., Tomisaka, K., \& Matsumoto, T. 2008, ApJ, 674, 997

Saslaw, W. C., \& Zipoy, D. 1967, Nature, 216, 976

Scannapieco, E., Schneider, R., \& Ferrara, A. 2003, ApJ, 589, 35

Schleicher, D. R. G., Banerjee, R., Sur, S., et al. 2010, A\&A, 522, A115

Schober, J., Schleicher, D. R. G., Federrath, C., et al. 2012, ApJ, 754, 99

Silk, J. 1977, ApJ, 211, 638

Smith, R. J., Glover, S. C. O., Clark, P. C., Greif, T., \& Klessen, R. S. 2011, MNRAS, 414, 3633
Sobral, D., Matthee, J., Darvish, B., et al. 2015, ApJ, 808, 139

Springel, V. 2005, MNRAS, 364, 1105

Springel, V. 2010, MNRAS, 401, 791

Stacy, A., Greif, T. H., \& Bromm, V. 2010, MNRAS, 403, 45

Stacy, A., Bromm, V., \& Loeb, A. 2011, MNRAS, 413, 543

Stacy, A., Greif, T. H., Klessen, R. S., Bromm, V., \& Loeb, A. 2013, MNRAS, 431,1470

Sterzik, M. F., Durisen, H., \& Zinnecker, H. 2003, A\&A, 411, 91

Sur, S., Schleicher D. R. G., Banerjee R., Federrath C., \& Klessen R. S. 2010, ApJ, 721, L134

Susa, H. 2013, ApJ, 773, 185

Susa, H., Uehara, H., Nishi, R., \& Yamada, M. 1998, Progr. Theor. Phys., 100, 63

Tegmark, M., Silk, J., Rees, M. J., et al. 1997, ApJ, 474, 1

Tornatore, L., Ferrara, A., \& Schneider, R. 2007, MNRAS, 382, 945

Turk, M. J., Abel, T., \& O'Shea, B. W. 2009, Science, 325, 601

Turk, M. J., Clark, P. C., Glover, S. C. O., et al. 2011, ApJ, 726

Whalen, D, Hueckstaedt, M., \& McConkie, O. 2010, ApJ, 712, 101

Wise, J. H., \& Abel, T. 2008, ApJ, 685, 40

Yoshida, N., Abel, T., Hernquist, L., \& Sugiyama, N. 2003, ApJ, 592, 645

Yoshida, N., Omukai, K., Hernquist, L., \& Abel, T. 2006, ApJ, 652

Yoshida, N., Omukai, K., \& Hernquist, L. 2008, Science, 321, 669 